\title{
ACCURATE MAPPING OF DATE PALMS AT DIFFERENT AGE-STAGES FOR THE PURPOSE OF ESTIMATING THEIR BIOMASS
}

\author{
S. M. Issa ${ }^{1, *}$, B. S. Dahy ${ }^{1}$, N. Saleous ${ }^{2}$ \\ ${ }^{1}$ Dept. of Geology, College of Science, UAEU, Al Ain, UAE - salem.essa@uaeu.ac.ae \\ ${ }^{2}$ Dept. of Geography, College of Humanities and Social Sciences, UAEU, Al Ain, UAE - nazmi.saleous@uaeu.ac.ae
}

\author{
Commission III, WG III/10
}

KEY WORDS: Carbon Stock, Date Palm, Hybrid Classification, Landsat 8 OLI, Mapping, WV-2

\begin{abstract}
:
The main objective of the current study was to produce an accurate map of date palm (DP) plantations in the Emirate of Abu Dhabi in United Arab Emirates (UAE) using moderate resolution Landsat-8 (OLI) imagery. The second objective was to be able to create a more detailed map depicting three different categories of date palms at three different age stages: young, medium, and mature. This was achieved using a hierarchical integrated approach: first, Landsat-8 OLI imagery were used to map mature date palms in the study area; second, an object oriented classification (OOC) approach was applied at the plantation level using sub-meter Worldview-2 imagery (WV-2) to depict and map medium and young aged palms. Three age-stage categories of date palms were mapped with acceptable accuracy. The primary outcome of this classification approach was the creation of detailed maps of date palms to be used as input to a remote sensing (RS) based biomass estimation model for the assessment of the above ground biomass (AGB) and carbon sequestered (CS) of date palms. Results were validated using existing ancillary data and field checks. DP were mapped with an overall accuracy of 94.5\% which was considered high in similar conditions of drylands, while the overall kappa statistics was estimated at 0.888 .
\end{abstract}

\section{INTRODUCTION}

Mapping vegetation for accurate measuring of biomass is a serious problem that must be resolved when quantifying carbon stock. Geospatial approach (RS/GIS) is widely used to collect information regarding forest $\mathrm{AGB}$ and vegetation structure as well as to monitor and map vegetation biomass and productivity at large scales(Iizuka and Tateishi, 2015; Makinde et al., 2017; Pflugmacher, 2011). Although the methods detailing vegetation cover mapping and estimation by RS and GIS are well developed, they face many challenges in certain ecosystems, such as arid lands ecosystems with high background contribution reflectance. Furthermore, many plant species cannot be easily distinguished from other targets due to their spectral similarities. The use of high spatial and spectral resolutions data has to be considered as they help resolve such ambiguities and play essential roles in the quality of land cover maps. Moreover, using satellite images to map and correlate environmental factors is only possible if the target vegetation spectra are strong enough to be identified within the pixel, which is the case only in some dryland environments(Aly et al., 2016; Tian et al., 2016). This latter requirement presents a major challenge in desert where vegetation is usually sparse, offering a small spectral target that requires higher resolutions to be detected(Bradley et al., 2019). Hyperspectral sensors showed plausible classification accuracies in mapping major forest species and predicting the susceptible areas of fruit malformation(Nagaraja, 2009). Hebbar et al., 2014, used LISS-IV data to classify fruit trees and found that old and mature plantations were classified more accurately while young and recently planted ones (3 years or less) showed poor classification accuracy due to mixed spectral signature, wider spacing and poor stands of plantations(Hebbar et al., 2014).

Furthermore, moderate resolution satellites (e.g. Landsat, and SPOT) proved to be effective in land cover classification for different research purposes and in different regions(Aly et al., 2016; Elhag, 2016; Rembold et al., 2000; Shaker et al., 2012). Such multispectral optical sensors have been widely utilized operationally in estimating and mapping AGB(Eisfelder et al., 2012; Kumar et al., 2015; Kumar and Mutanga, 2017; TSITSI, 2016; Vashum and Jayakumar, 2012). Indeed, moderate resolution satellite data offer plausible results after conducting specific approaches such as pan-sharpening or fusion techniques. Starting with Landsat 7, a panchromatic band with $15 \mathrm{~m}$ spatial resolution, that can be used to pan-sharpen other bands and hence increase their interpretability, was added to the already existing Landsat's multispectral sensors(Phiri and Morgenroth, 2017; Shaharum et al., 2018). Previous studies showed that such use of the panchromatic band helped achieve dramatic improvements (more than 15\%) in classification accuracies(Gilbertson et al., 2017).

The Landsat program, MSS, TM, ETM+ and the most recent Landsat-8 OLI, present unique advantages in land cover classification applications because: (1) it is the longest running uninterrupted Earth observation program since 1972; (2) its archives are the first to offer global images free of charge (free access approach since 2008)(Phiri and Morgenroth, 2017; Turner et al., 2015); (3) the current effects of climate change make the research on land cover classification methods based on the archived Landsat images an important resource(Barbosa et al., 2014; De Sy et al., 2012); (4) it is a very good source for vegetation change detection over large areas due to its relatively high temporal resolution (16-days revisit) and large swath (185 $\mathrm{km}$ ) and; (5) the suitability of the spatial resolution of Landsat series for regional mapping of biomass and carbon in a variety of ecosystems(Clerici et al., 2016). Baumann et al., 2018, found that Landsat-8 OLI is reliable for mapping woody vegetation (tree cover and shrub cover) in their study in Gran Chaco, south America. In their study for mapping tree canopy cover and AGB in woodlands landscape of Burkina Faso using Landsat-8 OLI, 
Karlson et al., 2015, found that the image texture is more correlated to tree cover attributes, in particular AGB, in open canopy conditions compared to closed canopies due to its ability to capture shadow structures caused by large trees(Karlson et al., 2015).

In the current study, a hybrid classification approach was adopted to create a classified map of the studied area producing seven different land use and land cover (LULC) classes as a first step. In the second step, an integration of our knowledge of the studied area within GIS environment has been employed to merge classes, producing a binary mask of only two categories: vegetation and non-vegetation. Lastly, high spatial resolution WV-2 imagery was used to identify and classify date palm plantations within the vegetation category.

\section{BACKGROUND: MAPPING DATE PALMS AT DIFFERENT AGE STAGES}

For the purpose of tree crown detection and delineation many algorithms were developed(Chepkochei, 2011; Hebbar et al., 2014; Lack and Bleisch, 2010; Rizvi et al., 2019; Sahay et al., 2017). However, results of tree detection and delineation can be affected by algorithm characteristics. Indeed, different approaches may give different results despite working in the same environment. Thus, it is important to select the appropriate algorithm to get the suitable results. Moreover, for any algorithm to work properly, crowns should be, at least, detectable and segmented as an object in the image before classification. Training areas of the classes that are to be extracted must be chosen very carefully to not include any background pixels and non-targeted classes based on visual analysis and on previous knowledge of the area by the interpreter. By using the pansharpened WV-2 images (spatial resolution 0.5 meter), date palm (DP) crowns can be differentiated from the background (soils, grasses, and weeds) and other shrubs and trees using colour, tone, texture, size and planting arrangement.

We used ERDAS IMAGINE 2020 Objective tool, from Hexagon Geospatial, that employs "feature models" which work on objects produced by image segmentation and various other pixel-based algorithms which, after being vectorized, can be processed by geometric and textural parameters(Lack and Bleisch, 2010). Several algorithms were used to extract and delineate tree crowns, however, the selection of the suitable one is a matter of user-defined parameters "cue metrics" and depend on trial and error (See Methodology section). In general, the steps can be divided to levels of analysis: (1) raster data analysis which includes identifying date palms from other vegetation and classifying their age stages (mature, medium, and young) according to their crowns, and (2) vectorising, cleaning up the vector layers and creating maps. In the raster analysis part, a pixel-based classifier relying on spectral, textural and site information, is used. Many studies chose Single feature probability (SFP) function to extract quantitatively the desired class(Chepkochei, 2011; Hebbar et al., 2014; Lack and Bleisch, 2010; Rizvi et al., 2019) using a Bayesian-classifier for statistic classification. Some studies include vegetation indices (e.g. NDVI) along with spectral and textural information(Chepkochei, 2011; Hebbar et al., 2014; Lack and Bleisch, 2010; Rizvi et al., 2019). In their study of mapping multiple horticulture crops using OOC in Krishna district in India, Sahay et. al., 2007 applied certain NDVI ranges as threshold to differentiate between mango trees and oil palms(Sahay et al., 2017). In the current study, in the UAE, both SFP function and NDVI threshold were used; the NDVI threshold was derived empirically from satellite imagery collected over the date palm samples identified in the field. An optimum range of NDVI values between 0.18 to 0.45 was found useful to differentiate between date palms and other vegetation types in the area. The definition of training areas for date palm crown as well as for background pixels is of central importance. Training areas are chosen carefully to not include any background pixel. Pixels of the individual palms (identified by training polygons) were submitted to compute pixel cue metrics to train the pixel classifier. The result is the pixel probability layer (Figure 1b). Then, the pixel probability layer is then converted into raster object using threshold and clump function. This function keeps only those pixels which have probability greater than or equal to a threshold value and assigns them the value of 1 , while the remaining pixels are assigned the value of 0 . Then, it performs a contiguity operation (clump) on the binary values and converts the pixel probability layer to a raster object layer (Figure 1c). For our study area, the threshold value was set to 0.50 based on the knowledge that lowering the threshold value can lead to inclusion of some non-tree pixels(Chepkochei, 2011; Hebbar et al., 2014; Rizvi et al., 2019). In order to separate and differentiate the palm crowns according to their ages, two other functions were applied: "Probability filter" and "Size filter" so that pixel objects with high probability are kept while others are given the status of 'background'. The "Probability filter" operator removes all the raster objects whose zonal probability mean is less than the specified minimum probability set to 0.75 in our case. While the "Size filter" removes raster objects that are too small or too large thus allowing one to restrict the set of raster objects to those of an appropriate crown size of individual palms(Chepkochei, 2011; Rizvi et al., 2019). With high resolution multispectral satellite imagery like WV-2, the differentiation between palms crown sizes according to their ages is explained in detail in the Methodology section below.

The second level of analysis was done on the vector data model by first vectorising the three raster outputs (mature, medium, and young), smoothing the polygons, calculating the areas, and cleaning up the maps manually (Figures 1d, 1e, and 1f).

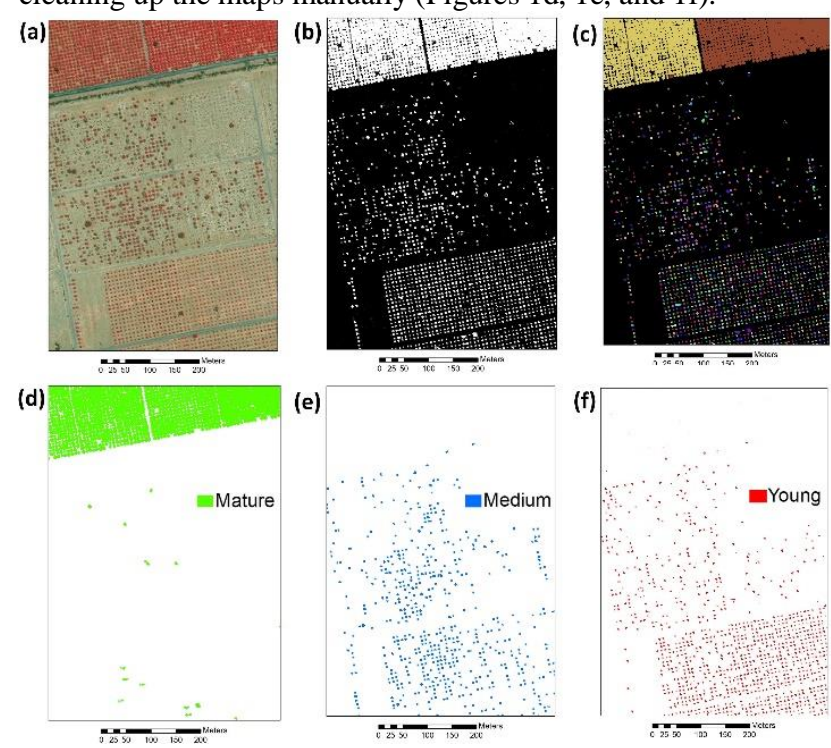

Figure 1. Separating age classes of date palm plantations: an example from $\mathrm{Al}$ Foah date palms farm: (a) Original WV-2 image (RGB:7,5,4); (b) SFP using Bayesian network; (c) Threshold and clump applied; (d) Mature palms layer; (e) Medium palms layer; and (f) Young palms layer. 


\section{METHODOLOGY}

Six Landat-8 OLI scenes covering Abu Dhabi emirate are preprocessed, pan-sharpened, and combined to build a single large mosaic image covering the study area. Each scene is classified using hybrid methods to produce an accurate LULC map, then reclassified (recoded) to produce a bitmap including only vegetation and non-vegetation areas. Then, a thematic map of date palm (DP) plantations in Abu Dhabi is built by distinguishing between DP and non-DP classes. An accuracy assessment procedure is run to evaluate the quality of the LULC map produced using available reference data and researchers' knowledge of the study area. On this basis, an error matrix is produced for each map to determine the overall accuracy, the user's and producer's accuracy, and the kappa coefficient(Rozenstein and Karnieli, 2011; Vicharnakorn et al., 2014). All procedures are implemented using ERDAS IMAGINE 2020 and ArcGIS 10.5 software packages. A detailed step-wise methodology is developed and presented in the following subsections.

\subsection{Study area}

The Emirate of Abu Dhabi is located in the West and South West part of UAE and is bounded by $22^{\circ} 55^{\prime}$ to $24^{\circ} 48^{\prime} \mathrm{N}$, and $51^{\circ} 30^{\prime}$ $\mathrm{E}$ to $56^{\circ} 00^{\prime} \mathrm{E}$. The desert areas constitute approximately $85 \%$ of the Emirate's main land, dividing into five landforms: sand dunes, inter-dunal sands, coastal sabkhas (flat plains with salty crust), inland sabkhas and exposed rock. The climate of the study area is affected by its location inside the desert with proximity to the coasts of the Arabian Gulf and the Gulf of Oman. The climate is characterized by a minimum average temperature of $14.7^{\circ} \mathrm{C}$ while the average maximum can reach around $42.9^{\circ} \mathrm{C}$. The annual average long-term rainfall is $59.1 \mathrm{~mm}$ and the humidity is 44.3\% (NCM, 1995-2018). Elevations vary between main sea level in coastal areas and increases smoothly in desert areas averaging an altitude of about $200 \mathrm{~m}$ above sea level (where most date palm plantations are found). The Emirate, which hosts the capital city of Abu Dhabi has witnessed dramatic conversions of its lands from being desert / desert-like covered country to an urbanized and well-developed modern state. The government invested heavily in planting and maintaining green areas, including more than 3000 farms many of which are date palm plantations. Six pan-sharpened images were used for building a single large mosaic image covering the whole Abu Dhabi area (Figure 2).

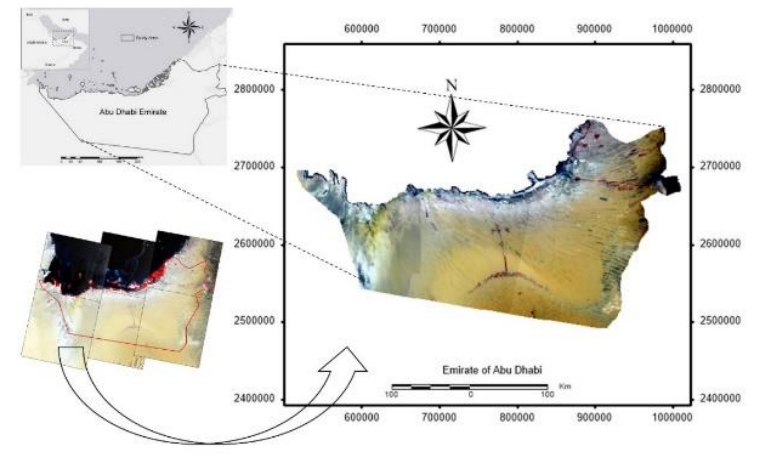

Figure 2. Study area (six Landsat-8 OLI images used to create one single mosaic image)

\subsection{Data processing}

Six cloud free Landat-8 OLI images covering the Emirate of Abu Dhabi (Path/Raw: 160/43, 160/44, 161/43, 161/44, 162/43 and 162/44), of Level-2 atmospherically corrected were downloaded from U.S. Geological Survey website (USGS). They all were acquired in April and May 2017. In addition, six panchromatic bands (level-1) were downloaded for the same scenes (same Path/Row and date) for pan-sharpening purposes. For each image selected, bands $2,3,4,5,6,7$, and 8 of Landsat-8 OLI were stacked and saved in ERDAS Imagine (.img) format. All images were co-registered to the Universal Transverse Mercator (UTM) projection (Zone 40, WGS 84). Furthermore, the panchromatic band- 8 was used to pan-sharpen the other bands in order to process the whole image sets at $15 \mathrm{~m}$ instead of the original multi spectral Landsat $30 \mathrm{~m}$ resolution. Nearest Neighbourhood (NN) algorithm was used for the resampling of the output images. The Anderson classification system (Level 1), recommended for use with Landsat resolution data(Anderson, 1976; Rozenstein and Karnieli, 2011), was used. Our classification scheme adopted seven LULC classes representing: urban (build-up including roads and buildings), vegetation (oasis, farms, palm plantations, parks, forests), sand sheets (dark soils and gravel), sand dunes (bright soils), deep water and shadows (including black surfaces), shallow water, and sabkhas (including bright surfaces). A hybrid classification, combining both supervised and unsupervised methods was applied. Therefore, two stages of classification were conducted; first, we ran unsupervised classification to minimize bias in the selection of training areas and seed signatures; second, a comprehensive set of spectral class signatures was used as training data for supervised classification(Rozenstein and Karnieli, 2011).

The six scenes were pan-sharpened and displayed as false color composite of bands 5,4, and 3; they were radiometrically adjusted using histogram equalization for better interpretation. Each image was initially clustered into 80 clusters using K-mean classifier and a maximum number of iterations of 80 , found to be the optimum number for Landsat data; allowing the clustering to stop naturally upon reaching the convergence threshold of 0.990 (Yang and Lo, 2002). Then, the images were classified using signatures from training sites that include all classes. The signatures were collected by digitizing polygons onto the images to collect the training samples (total of 720 training sets). The signatures were evaluated by drawing the mean signature values of each class against the Landsat-8 OLI bands. The maximum likelihood classification (MXL) was run and then, classes of the resulting image were recoded into the seven LULC classes. All classified scenes were mosaicked, and the study area was subset using available vector shapefile of the study area boundaries. The area of each LULC class was calculated in hectare. The thematic LULC map was filtered to clean up the single pixels by using a Majority Filter with window size of (3x3) and, manually cleaning up using the Fill Tool by filling the miss-classified pixels with the right values.

\subsection{Extraction of vegetation areas and mapping of date palm plantations}

3.3.1 Vegetated areas and date palms mapping (Landsat 8 OLI): In order to map date palm plantations in Abu Dhabi, a bit map was produced including only two land cover classes: vegetated / non-vegetated (Southworth et al., 2002). The Recode function in ERDAS Imagine 10.5 was used to produce the vegetation bitmap, where a value of 1 was assigned to vegetated class and a value of 0 was assigned to non-vegetated class. The same classification procedure used to produce the LULC map 
was applied here: an unsupervised classification of 80 clusters using k-mean classifier was run, followed by selecting training sets of pure spectral signatures to run MXL classifier; and then recoding to produce two classes: DP and non-DP classes. The selection of training sets (spectral signatures), was produced based on previous intensive field visits to date palm farms in the study area including: AlAin, AlFoah, Hayer, Yaher, Salamat, Khatem, Khaznah Wathbah, Nahdha, and Swihan. The GPS points $(\mathrm{x}, \mathrm{y})$ collected from these visits were projected on the image to collect pure spectral signatures of DP. Extraction of the vegetated areas required converting the LULC map of Abu Dhabi to a binary map by merging all non-vegetated classes namely: urban, sand sheets, sand dunes, deep water, shallow water, and sabkhas, into one class and be recoded as non-vegetation. Vegetated areas (pixels) were extracted (subset) from the original image of the study area by masking the non-vegetated pixels using Subset/Mask functions in ERDAS Imagine 10.5. Then, the vegetated areas layer was converted from raster to vector to produce vegetated areas in shapefile format. Here, a second level of classification was run in order to produce the date palm (DP) bitmap where a value of 1 is assigned to DP class and a value of 0 is assigned to non-DP class. Then, the areas of DP classes were computed (section 4).

3.3.2 Separating Age classes of date palm plantations (WV2): In this step, WV-2 covering all the vegetation areas of Abu Dhabi using the "vegetated area" shapefile which was created previously, was used to run OOC. The OOC procedure applied to $349 \mathrm{WV}-2$ images covering the eastern region of the Emirate of Abu Dhabi (Figure 3).

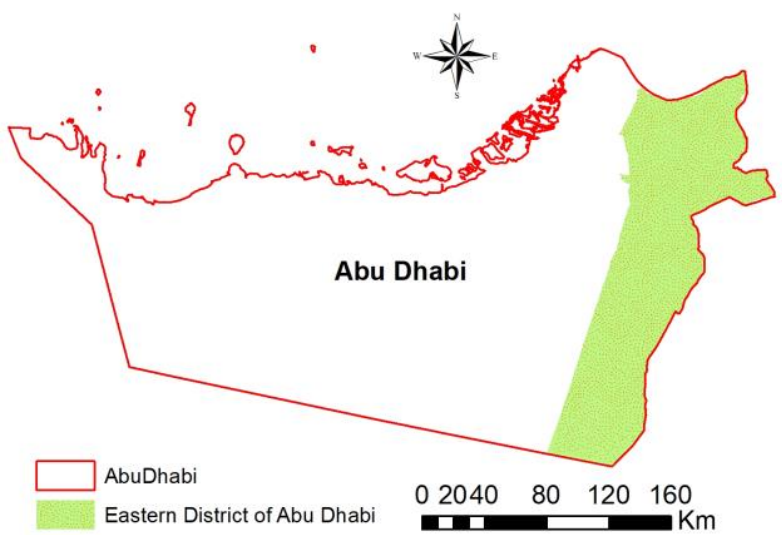

Figure 3. OOC was applied to WV-2 images at eastern district of Abu Dhabi to separate three different age stages of date palm plantations

WV-2 characterized by its multispectral bands (eight bands and one panchromatic) with $1.85 \mathrm{~m}$ spatial resolution for the eight bands and submeter $(0.46 \mathrm{~m})$ for the panchromatic. For each image selected, only three bands plus the panchromatic band of WV-2 were used and saved in ERDAS Imagine (.img) format. The three bands: band 3 (green), band 5 (red), and band 7 (NIR1) are equivalent to bands 3,4 , and 5 in Landsat 8 OLI (green, red, and NIR) which were proven to be significant RS variables in our study(Issa et al., 2019). The fusion of panchromatic with multispectral images of WV-2 scenes was done using the $\mathrm{NN}$ algorithm in order to create an image at a spatial resolution of $0.5 \mathrm{~m}$ (Jawak et al., 2013). In order to reduce the size of WV-2 images for further processing, the vegetated areas were subset from each WV-2 scene. The subset process was done by overlaying the vegetated area vector file produced in Landsat- 8
LULC classification and the personal interpretation of WV-2 images. A semi-automatic object-oriented feature extraction model was used for delineation of the date palm plantations using ERDAS Objective Imagine(Chepkochei, 2011; Hebbar et al., 2014; Lack and Bleisch, 2010; Rizvi et al., 2019).

The feature model tree for extraction date palm crowns was created and optimized for a small area in AlFoah date palms farm, north to AlAin city. This farm has more than 60,000 palms in different cultivars and ages and was intensively visited during the project(Issa et al., 2019, 2018). Later, the feature model tree was transferred to a whole WV-2 pan-sharpened images(Lack and Bleisch, 2010). Finally, the optimal parameters were set using the trial-and-error approach.

Finally, the shapefiles were merged using geoprocessing tool (Merge) in ArcGIS software and map was produced for mature, medium, and young date palm plantations. The area of each DP age stage was computed. A confusion matrix was created, and accuracy measures were calculated for LULC map which produced by applying pixel-based classification (PBC) on Landsat 8 OLI images and the age stages map of date palms which produced by applying OOC on WV-2 images.

\section{RESULTS}

Figure 4 shows the main classes' percentage in the study area including: deep water, shallow water, urban, vegetation, sand dunes, sand sheets, and sabkhas.

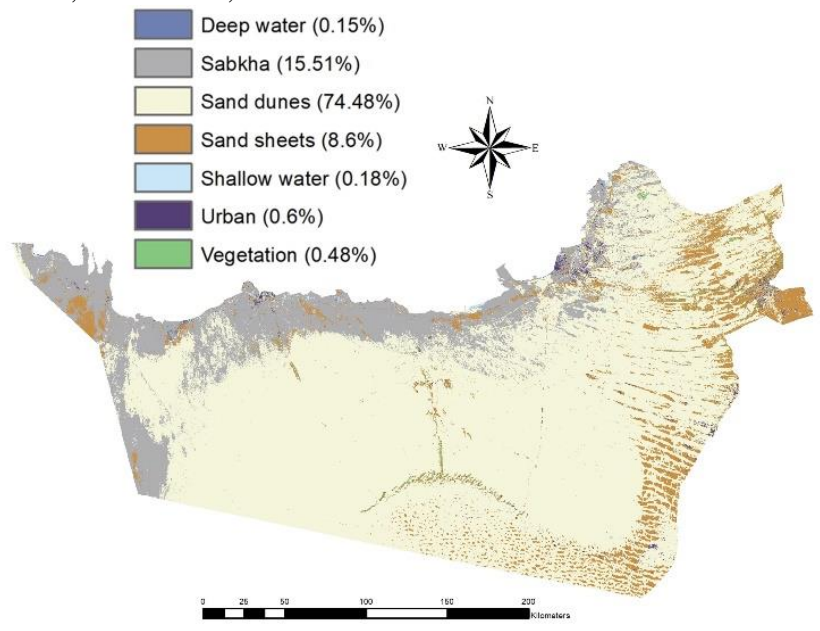

Figure 4. LULC map of the study area

As mentioned in the Methodology section, the Recode function in ERDAS IMAGINE 2020 was used to produce the DP bitmap where a value of 1 is assigned to DP class and a value of 0 is assigned to non-DP class (Figure 5). It was concluded that young and medium date plantations $(\leq 10$ years) were difficult to differentiate due to soil background. Therefore, date palm plantations extracted from Landsat 8 OLI images refer to mature date palm plantations only ( $\geq 10$ years) with an average crown diameter passing 5 meters and average total height around 6.6 meter. For overestimated or underestimated classes, the problem was solved manually by working on the vector polygons and the removal of erroneous pixels benefiting from our knowledge of the study area and existing reference maps using the Fill tool in ERDAS imagine 10.5 to fill the miss-classified pixels with the right values. 


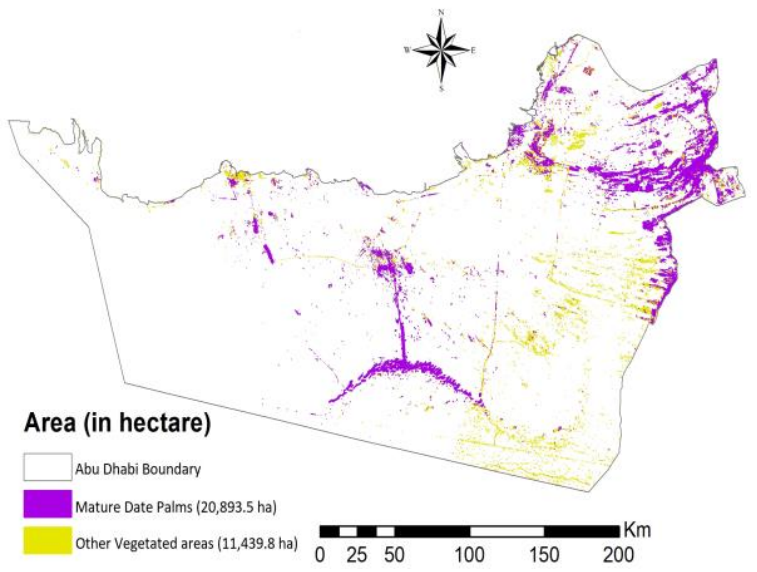

Figure 5. Vegetation bitmap and derived mature date palm plantations in the study area using Landsat 8 OLI (PBC method)

The evaluation of spectral signatures derived from the 740 training sets showed that the short wave infrared (SWIR1\& SWIR2 bands) offered the best separablity power among all Landsat 8 OLI bands. However, some combinations were found to be more efficient in identifying and mapping the vegetation class such as: (RED, SWIRE1, SWIRE2) or (RED, GREEN, SWIR1) or (RED, GREEN, SWIR2) or (GREEN, SWIR1, SWIR2). Table 1 shows percentages and areas (in hectare) of each class of the LULC map of the study area (Emirate of Abu Dhabi), where sand dunes occupied around two third of the emirate with around five million hectares. Sabkhas, sand with high level of salts, occupied $15.51 \%$ of the emirate with more than one million hectares while the sand sheets including gravels occupied $8.6 \%$ of the whole area with more than half million hectares. Urban areas occupied $0.6 \%$ with $40,102.6$ hectares while vegetated areas occupied $0.49 \%$ of the total area of the emirate with more than thirty thousand hectares.

\begin{tabular}{|l|c|c|}
\hline LULC & Area (ha) & Percentage (\%) \\
\hline Deep water & $9,677.7$ & 0.15 \\
Shallow water & $11,847.2$ & 0.18 \\
Urban & $40,102.6$ & 0.6 \\
Vegetation & $32,333.3$ & 0.49 \\
Sand dunes & $4,957,180$ & 74.48 \\
Sand sheets & 572,665 & 8.6 \\
Sabkhas & $1,032,170$ & 15.51 \\
Total & $6,655,975.8$ & - \\
\hline
\end{tabular}

Table 1. Areas and percentages of the study area LULC map classes

Table 2 shows the area (in hectare) of the mature date palm category of the study area, which occupied $64.62 \%$ of the vegetated areas in the emirate.

\begin{tabular}{|l|c|}
\hline Class & Area (ha) \\
\hline Mature Date Palm Plantations (> 10 years) & $20,893.5$ \\
Other vegetated areas & $11,439.8$ \\
\hline
\end{tabular}

Table 2. Areas in ha, of mature date palm class and other vegetated areas in Abu Dhabi

Finally, Date Palms at different age classes of the Eastern District of Abu Dhabi was mapped with high accuracy (Figure 6).

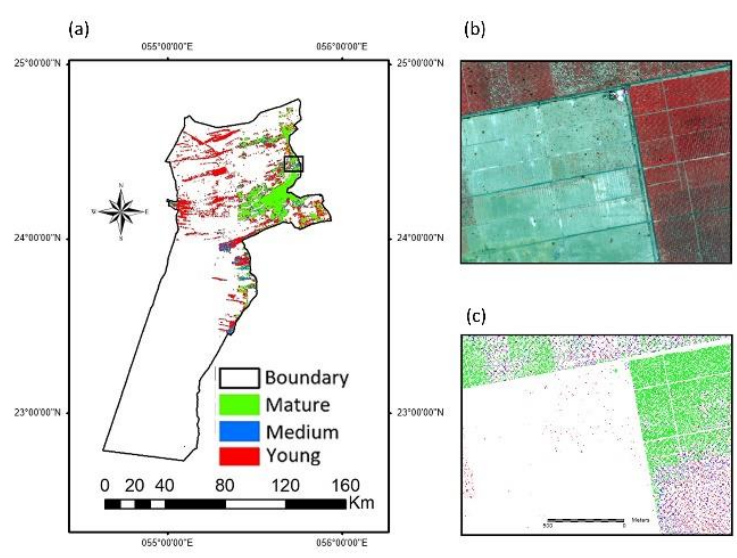

Figure 6. Map of Date Palms age classes at Eastern District, Abu Dhabi (b) AlFoah date palms farm in false color image (c) Zoom map of date palm age classes

Table 3 shows the areas (in hectare) of the three age stages of date palms in Eastern District of Abu Dhabi.

\begin{tabular}{|l|c|}
\hline Class & Area (ha) \\
\hline Mature Date Palms & 1565.03 \\
Medium Date Palms & 959.01 \\
Young Date Palms & 316.98 \\
Total & $2,841.02$ \\
\hline
\end{tabular}

Table 3. Area per hectare of date palms in Eastern District, Abu Dhabi

\section{ACCURACY ASSESSMENT}

The accuracy of the classified maps was assessed by a set of points sampled using the stratified random sampling of 350 points, 50 for each class. The points, different from the training points, were randomly selected for each of the classes using ERDAS Imagine' accuracy assessment tool. In addition, we used Google Earth, reference map and personal knowledge of the area in this process. A confusion matrix was created, and accuracy measures were calculated for the seven classes included in the LULC map. The results summarized in (Table 4) indicate a good overall performance of the classification with a Kappa value of about $81 \%$.

\begin{tabular}{|l|c|c|c|c|c|c|c|c|c|c|}
\hline Class & DW & SW & U & V & SD & SS & SB & Total & $\begin{array}{c}\text { PA } \\
(\%)\end{array}$ & $\begin{array}{c}\text { UA } \\
(\%)\end{array}$ \\
\hline DW & 38 & 1 & 0 & 0 & 0 & 3 & 8 & 50 & 92.7 & 76 \\
SW & 2 & 44 & 1 & 0 & 1 & 0 & 2 & 50 & 95.6 & 88 \\
U & 0 & 0 & 36 & 7 & 0 & 7 & 0 & 50 & 85.7 & 72 \\
V & 0 & 1 & 4 & 40 & 2 & 1 & 2 & 50 & 74.1 & 80 \\
SD & 0 & 0 & 0 & 1 & 45 & 2 & 2 & 50 & 81.8 & 90 \\
SS & 0 & 0 & 1 & 6 & 2 & 40 & 1 & 50 & 74.1 & 80 \\
SB & 1 & 0 & 0 & 0 & 5 & 1 & 43 & 50 & 74.1 & 86 \\
Total & 41 & 46 & 42 & 54 & 55 & 54 & 58 & 350 & & \\
OA (\%) & 81.7 & & &
\end{tabular}

SS: Sand Sheets; SB: Sabkhas;

PA: Producer's Accuracy; UA: User's Accuracy, OA: Overall Accuracy

Table 4. Accuracy assessment of the LULC map

Accuracy of the Date Palm classification is summarized in table 5 where high accuracy with an overall accuracy of $94.5 \%$ and a Kappa coefficient of $88 \%$ were achieved. 


\begin{tabular}{|l|c|c|c|c|c|}
\hline Class name & $\begin{array}{c}\text { Date } \\
\text { Palms }\end{array}$ & Others & Total & $\begin{array}{c}\text { Producer } \\
\text { Accuracy }\end{array}$ & $\begin{array}{c}\text { User } \\
\text { Accuracy }\end{array}$ \\
\hline Date Palms & 105 & 6 & 111 & $95.4 \%$ & $94.6 \%$ \\
Others & 5 & 84 & 89 & $93.3 \%$ & $94.4 \%$ \\
Total & 110 & 90 & 200 & & \\
Overall Accuracy & $94.5 \%$ & & & & \\
Kappa & 0.888 & & & & \\
\hline
\end{tabular}

Table 5. Accuracy assessment of mapping the date palms using OOC on WV-2 images

\section{DISCUSSION}

Mapping specific vegetation species with medium resolution satellite imagery, such as Landsat-8 OLI, was a great challenge. Hybrid classification approach combining supervised and unsupervised algorithms was successful in identifying and mapping mature date palm plantations in the current study. On the other hand, delineating young date palms was a little harder to achieve requiring direct (manual) input from the researcher. Alternatively, a more detailed satellite imagery with sub-meter resolution such as WV-2 was used to medium and young date palms.

The same classification method used to produce LULC map was applied to produce DP map. The selection of training sets (spectral signatures), were produced based on previous intensive field visits to date palm farms in the study area. The date palm age and plots densities had an effect on the estimation of AGB. Moreover, some plots had sparse distribution of date palms with no regular spacing system due to human disturbances.

Examining the mean spectral signatures of LULC classes derived from Landsat- 8 data showed that vegetation was well separated from deep water in the NIR and from all other classes in the red and green bands although the red band was better separator than the green band. In conclusion, and based on the researchers' experiments, the following combinations were the most helpful in detecting the vegetation class and producing the final bitmap:

1. RED, SWIR1, SWIR2, OR

2. RED, GREEN, SWIR1, OR

3. RED, GREEN, SWIR2, OR

4. GREEN, SWIR1, SWIR2

The produced LULC map showed that sand dunes occupied around two third of the emirate with around five million hectares. Sabkhas and sand, with high level of salts, occupied $15.51 \%$ of the study area with more than one million hectares while the sand sheets including gravels occupied $8.6 \%$ of the whole area with more than half million hectares. Urban areas occupied $0.6 \%$ with $40,102.6$ hectares while vegetated areas occupied $0.49 \%$ of the total area with more than thirty thousand hectares. The area (in hectare) of the mature date palm plantations of the study area occupied $64.62 \%$ of the vegetated areas in the emirate of Abu Dhabi.

WV-2 covering the areas occupied by DP and identified by the classification of Landsat 8 data were used to perform an OOC to map medium and young age DP. The overall map of DP comprising all age classes was then produced with a high accuracy for the Emirate.

\section{CONCLUSIONS \& RECOMMENDATIONS}

In the current study, we mapped LULC classes in the Emirate of Abu Dhabi using medium scale Landsat- 8 OLI imagery. We adopted a hybrid classification approach combining both supervised and unsupervised classifications to identify and map vegetation class in order to extract date palm (DP) plantations. The adopted approach was very successful in mapping mature date palm plantations while the results with young palm were more challenging due to their sparse structure bringing more soil background contribution to the DP signatures. To overcome this problem, we adopted an OOC approach using high resolution WV-2 imagery to identify and map medium and young DP. The fusion of moderate spatial resolution Landsat 8 with high spatial resolution WV-2 imagery, for creating detailed maps of DP at different age stages, proved to be successful and appropriate for creating DP maps that can feed a RS-based model for AGB estimation.

Finally, date palm plantations were mapped with an overall accuracy of $81.71 \%$ which is considered high in similar conditions of drylands, while the overall kappa statistics was estimated at $80.94 \%$. Furthermore, the accurate separation of date palms into 3 age categories allowed for a better estimation of their AGB and the carbon they sequester. The maps created in this study made possible to apply a non-destructive approach for estimating AGB and carbon sequestered in DP in the arid environment of UAE. The approach can easily be extended to larger areas in the region.

\section{ACKNOWLEDGMENTS}

We would like to express our appreciation to the Research Affairs - United Arab Emirates University for their financial support under fund grant \# 31S247.

\section{REFERENCES}

Aly, A.A., Al-Omran, A.M., Sallam, A.S., Al-Wabel, M.I., AlShayaa, M.S., 2016. Vegetation cover change detection and assessment in arid environment using multi-temporal remote sensing images and ecosystem management approach. Solid Earth 7, 713-725.

Anderson, J.R., 1976. A land use and land cover classification system for use with remote sensor data. US Government Printing Office.

Barbosa, J.M., Broadbent, E.N., Bitencourt, M.D., 2014. Remote sensing of aboveground biomass in tropical secondary forests: A review. International Journal of Forestry Research 2014.

Bradley, A.V., Haughan, A.E., Al-Dughairi, A., McLaren, S.J., 2019. Spatial variability in shrub vegetation across dune forms in central Saudi Arabia. Journal of Arid Environments 161, 72-84.

Chepkochei, L.C., 2011. Object-oriented image classification of individual trees using Erdas Imagine objective: case study of Wanjohi area, Lake Naivasha Basin, Kenya, in: Proceedings of the Kenya Geothermal Conference, Nairobi, Kenya.

Clerici, N., Rubiano, K., Abd-Elrahman, A., Posada Hoestettler, J.M., Escobedo, F.J., 2016. Estimating aboveground biomass and carbon stocks in periurban Andean secondary forests using very high resolution imagery. Forests 7, 138.

De Sy, V., Herold, M., Achard, F., Asner, G.P., Held, A., Kellndorfer, J., Verbesselt, J., 2012. Synergies of multiple remote sensing data sources for REDD+ monitoring. Current Opinion in Environmental Sustainability 4, 696-706. 
Eisfelder, C., Kuenzer, C., Dech, S., 2012. Derivation of biomass information for semi-arid areas using remote-sensing data. International Journal of Remote Sensing 33, 2937-2984.

Elhag, M., 2016. Evaluation of different soil salinity mapping using remote sensing techniques in arid ecosystems, Saudi Arabia. Journal of Sensors 2016.

Gilbertson, J.K., Kemp, J., Van Niekerk, A., 2017. Effect of pansharpening multi-temporal Landsat 8 imagery for crop type differentiation using different classification techniques. Computers and electronics in agriculture 134, 151-159.

Hebbar, R., Ravishankar, H.M., Subramoniam, S.R., Uday, R., Dadhwal, V.K., 2014. Object oriented classification of high resolution data for inventory of horticultural crops. The International Archives of Photogrammetry, Remote Sensing and Spatial Information Sciences 40, 745.

Iizuka, K., Tateishi, R., 2015. Estimation of CO2 sequestration by the forests in Japan by discriminating precise tree age category using remote sensing techniques. Remote Sensing 7, 1508215113.

Issa, S., Dahy, B., Ksiksi, T., Saleous, N., 2018. Development of a new allometric equation correlated with RS variables for the assessment of date palm biomass.in: Proceedings of Asian conference of remote sensing (ACRS2018), KL, Malaysia.

Issa, S., Dahy, B., Saleous, N., Ksiksi, T., 2019. Carbon stock assessment of date palm using remote sensing coupled with fieldbased measurements in Abu Dhabi (United Arab Emirates). International Journal of Remote Sensing 0, 1-20.

Jawak, S.D., Luis, A.J., Panditrao, S.N., Khopkar, P.S., Jadhav, P.S., 2013. Advancement in landcover classification using very high resolution remotely sensed 8-band WorldView-2 satellite data. International Journal of Earth Sciences and Engineering 6, $1742-1749$.

Karlson, M., Ostwald, M., Reese, H., Sanou, J., Tankoano, B., Mattsson, E., 2015. Mapping tree canopy cover and aboveground biomass in Sudano-Sahelian woodlands using Landsat 8 and random forest. Remote Sensing 7, 10017-10041.

Kumar, L., Mutanga, O., 2017. Remote Sensing of AboveGround Biomass. Remote Sensing 9, 935.

Kumar, L., Sinha, P., Taylor, S., Alqurashi, A.F., 2015. Review of the use of remote sensing for biomass estimation to support renewable energy generation. Journal of Applied Remote Sensing 9, 097696-097696.

Lack, N., Bleisch, S., 2010. Object-based change detecion for a cultural-historical survey of the landscape-from cow trails to walking paths. The International Archives of the Photogrammetry, Remote Sensing and Spatial Information Sciences 38.

Makinde, E.O., Womiloju, A.A., Ogundeko, M.O., 2017. The geospatial modelling of carbon sequestration in Oluwa Forest, Ondo State, Nigeria. European Journal of Remote Sensing 50, 397-413.

Nagaraja, A., 2009. Predicting susceptible areas of mango malformation through remote sensing and GIS (PhD Thesis).
Pflugmacher, D., 2011. Remote sensing of forest biomass dynamics using Landsat-derived disturbance and recovery history and lidar data. Oregon State University.

Phiri, D., Morgenroth, J., 2017. Developments in Landsat land cover classification methods: A review. Remote Sensing 9, 967.

Rembold, F., Carnicelli, S., Nori, M., Ferrari, G.A., 2000. Use of aerial photographs, Landsat TM imagery and multidisciplinary field survey for land-cover change analysis in the lakes region (Ethiopia). International Journal of Applied Earth Observation and Geoinformation 2, 181-189.

Rizvi, R.H., Newaj, R., Srivastava, S., Yadav, M., 2019. Mapping tress on farmlands using OBIA method and high resolution satellite data: a case study of Koraput district, Odisha. ISPRS - International Archives of the Photogrammetry, Remote Sensing and Spatial Information Sciences XLII-3/W6, 617-621.

Rozenstein, O., Karnieli, A., 2011. Comparison of methods for land-use classification incorporating remote sensing and GIS inputs. Applied Geography 31, 533-544.

Sahay, B., Chakraborty, A., Chaudhary, K.K., Laxman, B., Murthy, C., Rao, P., 2017. Mapping Multiple Horticulture Crops using Object Oriented Classification Techniques 9.

Shaharum, N.S.N., Shafri, H.Z.M., Gambo, J., Abidin, F.A.Z., 2018. Mapping of Krau Wildlife Reserve (KWR) protected area using Landsat 8 and supervised classification algorithms. Remote Sensing Applications: Society and Environment 10, 24-35.

Shaker, A., Yan, W.Y., El-Ashmawy, N., 2012. Panchromatic satellite image classification for flood hazard assessment. Journal of applied research and technology 10, 902-911.

Southworth, J., Nagendra, H., Tucker, C., 2002. Fragmentation of a landscape: Incorporating landscape metrics into satellite analyses of land-cover change. Landscape Research 27, 253 269.

Tian, F., Brandt, M., Liu, Y.Y., Verger, A., Tagesson, T., Diouf, A.A., Rasmussen, K., Mbow, C., Wang, Y., Fensholt, R., 2016. Evaluating vegetation optical depth (VOD) using AVHRR NDVI and in situ green biomass data over West African Sahel. Remote Sensing of Environment 177, 265-276.

TSITSI, B., 2016. Remote sensing of aboveground forest biomass: A review. Tropical Ecology 57, 125-132.

Turner, W., Rondinini, C., Pettorelli, N., Mora, B., Leidner, A.K., Szantoi, Z., Buchanan, G., Dech, S., Dwyer, J., Herold, M., 2015. Free and open-access satellite data are key to biodiversity conservation. Biological Conservation 182, 173-176.

Vashum, K.T., Jayakumar, S., 2012. Methods to estimate aboveground biomass and carbon stock in natural forests-A review. $J$. Ecosyst. Ecogr 2, 1-7.

Vicharnakorn, P., Shrestha, R.P., Nagai, M., Salam, A.P., Kiratiprayoon, S., 2014. Carbon stock assessment using remote sensing and forest inventory data in Savannakhet, Lao PDR. Remote Sensing 6, 5452-5479.

Yang, X., Lo, C.P., 2002. Using A time series of satellite imagery ato detect land use and cover changes in Atlanta, Georgia. International Journal of Remote Sensing 23, 1775-1798. 